\title{
A NOTE ON PERIODIC POINTS \\ OF EXPANDING MAPS OF THE INTERVAL
}

\author{
BAU-SEN DU
}

\begin{abstract}
We sharpen a result of Byers on the existence of periodic points for some continuous expanding maps of the interval and generalize it to some classes of continuous maps of the interval which are not necessarily expanding. We then use these results to construct one-parameter families of continuous maps of the interval which have a bifurcation from fixed points directly to period 3 points together with a series of reverse bifurcations from period 3 points back to fixed points. Consequently, our results also provide examples of one-parameter families of continuous maps of the interval whose topological entropy jumps from zero to some positive number and then changes back to zero as the parameter varies.
\end{abstract}

\section{Introduction}

Let $I$ denote the closed unit interval $[0,1]$ and let $C^{0}(I, I)$ denote the space of all continuous maps from $I$ into itself. We call a piecewise monotonic map $\tau$ in $C^{0}(I, I)$ expanding if there exists a constant $\lambda>1$ such that $|\tau(x)-\tau(y)| \geq \lambda|x-y|$ whenever both $x$ and $y$ belong to some interval on which $\tau$ is monotonic and call $\lambda$ an expanding constant for $\tau$. It is obvious that if $\tau \in C^{0}(I, I)$ is expanding with

Received 23 August 1985.

Copyright Clearance Centre, Inc. Serial-fee code: 0004-9727/86 $\$ A 2.00+0.00$. 
expanding constant $\lambda>1$, then, for every positive integer $m, \tau^{m}$ is also expanding, but with $\lambda^{m}$ as an expanding constant.

In [2], it is shown that if $\tau \in C^{0}(I, I)$ is an expanding map with expanding constant $\lambda>1$, which is increasing on $[0, c]$ and decreasing on $[c, 1]$, and if $2^{1 / 2^{n}} \leq \lambda$ for some nonnegative integer $n$, then $\tau$ has a periodic point of least period $2^{m} \cdot 3$ for some nonnegative integer $m \leq n$. In this note, we first sharpen the above result by showing that the condition $2^{1 / 2^{n}} \leq \lambda$ can be replaced by the condition $[(1+\sqrt{5}) / 2]^{1 / 2^{n}} \leq \lambda$ which is best in the sense that we cannot have the same conclusion if the number $(1+\sqrt{5}) / 2$ is replaced by any smaller positive number (see Theorem 1). We also generalize this new result to some classes of continuous maps in $C^{0}(I, I)$ which are not necessarily expanding (see Theorems 2 and 3 ). We then indicate how to use these results to construct one-parameter families of continuous maps in $C^{0}(I, I)$ which have a bifurcation from fixed points directly to periodic points of least period $2^{k} \cdot 3$ for some nonnegative integer $k$ (together with a series of reverse bifurcations back to fixed points) (see Corollaries 4 and 5).

As is well-known, continuous maps in $C^{0}(I, I)$ without periodic points other than fixed points have very simple dynamics $([3],[4$, Theorem 2]) and those in $C^{0}(I, I)$ with periodic points of least period not an integral power of 2 have an uncountable scrambled set [8] on which the dynamics is extremely complicated (see also [5]). Therefore, our results provide examples of one-parameter families of continuous maps in $C^{0}(I, I)$ which have a bifurcation from very simple dynamics directly to chaos. It is also well-known that continuous maps in $C^{0}(I, I)$ without periodic points of least period $2^{m} \cdot n$ for any integer $m \geq 0$ and any odd integer $n>1$ have zero topological entropy [11] and those in $C^{0}(I, I)$ with periodic points of least period $2^{m} \cdot n$ for some integer $m \geq 0$ and some odd integer $n>1$ have positive topological entropy ([1], [7]). Consequently, our results also provide examples of one-parameter families of continuous maps in $C^{0}(I, I)$ whose topological entropy jumps from zero to some positive number and then changes back to zero as the parameter varies. 
In Section 2, we state our main results (Theorems 1, 2, and 3) and present two applications of these results (Corollaries 4 and 5). The proofs of Theorems 1, 2, and 3 will appear in Sections 3, 4, and 5 respectively.

\section{Statement of main results}

In this section, we state our main results and present two applications of these results.

THEOREM 1. Let $\tau \in C^{0}(I, I)$ be con expanding map with expanding constant $\lambda>1$, which is increasing on $[0, c]$ and decreasing on $[c, 1]$. If $[(1+\sqrt{5}) / 2]^{1 / 2^{n}} \leq \lambda$ for some nonnegative integer $n$, then $\tau$ has a periodic point of least period $2^{m} \cdot 3$ in $[c, 1]$ for some nonnegative integer $m \leq n$.

Remark 1. The condition $[(1+\sqrt{5}) / 2]^{1 / 2^{n}} \leq \lambda$ in the above theorem is best in the sense that we cannot have the same conclusion if the number $(1+\sqrt{5}) / 2$ is replaced by any smaller positive number [9, Theorem 2.2].

Remark 2. In light of the above theorem and Theorem 2.5 in [1], we pose the following question: For every positive integer $k$, let $\lambda_{k}$ denote the largest zero of the polynomial $x^{2 k+1}-2 x^{2 k-1}-1$. Let $\tau \in C^{0}(I, I)$ be an expanding map, with expanding constant $\lambda>1$, which is increasing on $[0, c]$ and decreasing on $[c, 1]$. If $\left(\lambda_{k}\right)^{1 / 2^{n}} \leq \lambda$ for some positive integer $k$ and some nonnegative integer $n$, must $\tau$ have a periodic point of least period $2^{m} \cdot(2 k+1)$ in $[c, 1]$ for some nonnegative integer $m \leq n$ ?

Remark 3. For expanding maps with more than two monotonic pieces, the conclusion of Theorem 1 may not hold as shown by the following example:

EXAMPLE. For every positive integer $n$, let $f_{n}(x)$ be the continuous map from $[0,1]$ into itself defined by

(i) $f_{n}\left(i / 2^{n+1}\right)=1 / 2$ for all even $i$ with $0 \leq i \leq 2^{n+1}$;

(ii) $f_{n}\left(i / 2^{n+1}\right)=1$ for all odd $i$ with $0<i<2^{n}$;

(iii) $f_{n}\left(i / 2^{n+1}\right)=0$ for all oda $i$ with $2^{n}<i<2^{n+1}$;

(iv) $f_{n}(x)$ is linear on each component of the complement of the set 
$\left\{i / 2^{n+1} \mid i=0,1,2, \ldots, 2^{n+1}\right\}$ in $[0,1]$

Then $f_{n}(x)$ has the number $2^{n}$ as an expanding constant, but it only has periodic points of even periods and no periodic points of odd periods other than fixed points.

In the following, we present a result which generalizes Theorem 1 to some class of continuous maps in $C^{0}(I, I)$ which are not necessarily expanding.

THEOREM 2. Let $\delta, \lambda_{1}, \lambda_{2}$, and $\lambda_{3}$ be ony four fixed numbers with $0<\delta<1 / 2, \lambda_{1}>0, \lambda_{2}>0$, and $\lambda_{3}>0$. Assume that $f(x)$ is a continuous map in $C^{0}(I, I)$ which satisfies the following six conditions:

(i) $f(1 / 2)=1$;

(i.i) $1 / 2-\delta \leq f(1)<1 / 2$;

(iii) $[f(x)-f(y)] /(x-y) \geq \lambda_{1}$ for alz $1 / 2-\delta \leq y<x \leq 1 / 2$;

(iv) $[f(x)-f(y)] /(x-y) \leq-\lambda_{2}$ for alz $1 / 2 \leq y<x \leq 1 / 2+\delta$;

(v) $[f(x)-f(y)] /(x-y) \leq-\lambda_{3}$ for alZ $1-\delta \leq y<x \leq 1$;

(vi) The equation $f(x)=1 / 2$ has a solution in $(1-\delta, 1)$. If the equation $f(x)=1 / 2+\delta$ has a (unique) solution in $[1-\delta, 1]$, let $x_{0}$ denote the solution. Otherwise, let $x_{0}=1-\delta$. Then the following hold.

(1) If $f^{2}(1) \geq x_{0}$ and $\min \left\{\lambda_{1} \lambda_{3}, \lambda_{2} \lambda_{3}\right\} \geq[(1+\sqrt{5}) / 2]^{1 / 2^{n}}$ for some integer $n \geq 0$, then $f(x)$ has a periodic point of least period $2^{n+1} \cdot 3$ in $\left[f^{2}(1), 1\right]$.

(2) If $f^{2}(1) \geq x_{0}$ and $\min \left\{\lambda_{1}^{2} \lambda_{3}^{2}, \lambda_{1} \lambda_{2} \lambda_{3}^{2}\right\} \geq[(1+\sqrt{5}) / 2]^{1 / 2}{ }^{n}$ for some integer $n \geq 0$, then $f(x)$ has a periodic point of least period $2^{n+2} \cdot 3$ in $\left[f^{2}(1), 1\right]$.

Note. If, in the above theorem, Condition (ii) is replaced by (ii)' $1 / 2<f(1) \leq 1 / 2+\delta$ and condition $(v)$ is replaced by (v)' $[f(x)-f(y)] /(x-y) \geq \lambda_{3}$ for all $1-\delta \leq y<x \leq 1$, then a similar conclusion holds.

In the above theorem, if $\lambda_{1}$ is large enough (for example if $\lambda_{1} \geq 2$ ), then the best possible case of the existence of a periodic point of least 
period 6 in $\left[f^{2}(1), 1\right]$ can be guaranteed to occur under somewhat weaker conditions.

THEOREM 3. Let $\delta$ and $B$ be two fixed numbers with $0<\delta<1 / 2$ and $(1-\delta) / 2 \leq \beta<1 / 2$. Assume that $f(x)$ is a continuous mop in $C^{0}(I, I)$ which satisfies the following five conditions:

(i) $f(1 / 2)=1$ and $f(1)=B$;

(ii) $1-\delta \leq f(\beta), f^{2}(\beta) \leq 1 / 2+\delta$, and $1-\delta \leq f^{3}(\beta)$;

(iii) $f(x) \leq 2 x$ for alz $1 / 2-\delta \leq x \leq 1 / 2$;

(iv) $f(x) \leq-x+3 / 2$ for all $1 / 2 \leq x \leq 1 / 2+\delta$;

(v) $f(x) \geq-x+1+\beta$ for all $1-\delta \leq x \leq 1$.

Then $f(x)$ has a periodic orbit of least period 6 whose intersection with each of the intervals $(\beta, 1 / 2)$ and $\left(1 / 2, f^{2}(\beta)\right)$ is not empty.

Remark 4. It is easy to see that, under the conditions as stated in the above theorem, $f(x)$ does not necessarily have periodic points of odd periods in $(\beta, 1 / 2)$ or in $\left(1 / 2, f^{2}(\beta)\right)$ other than fixed points.

Remark 5. Theorems 2 and 3 are useful for the purpose of constructing simple examples of one-parameter families of continuous maps in $C^{0}(I, I)$ which have a bifurcation from fixed points directly to periodic points of least period $2^{m} \cdot 3$ for some fixed integer $m \geq 0$ (together with a series of reverse bifurcations from period $2^{m} \cdot 3$ points back to fixed points). In the following, we present two such examples. They are all easy consequences of Theorem 2. Similar applications of Theorem 3 are obvious and omitted.

COROLLARY 4 . Let $\delta, \lambda_{1}, \lambda_{2}$, and $\lambda_{3}$ be four fixed positive numbers with $0<\delta<1 / 2$ and let $F(\alpha, x)$ be a continuous map from $I \times I$ into $I$. Let $f_{\alpha}(x)$ be the one-parameter family (with $\alpha$ as the parameter) of continuous maps in $C^{0}(I, I)$ defined by $f_{\alpha}(x)=F(\alpha, x)$ for $0 \leq x \leq 1$ and $0 \leq \alpha \leq 1$. Assume that, for every $\alpha$ with $1 / 2<\alpha<1 / 2+\delta$, there exists a point $c_{\alpha}$ in $(1 / 2-\delta, 1 / 2+\delta)$ which varies continuously with $\alpha$ and $c_{1 / 2}=1 / 2$ such that $f_{\alpha}(x)$ satisfies 
the following five conditions:
(i) $1-\alpha \leq f_{\alpha}(1)<c_{\alpha}$
(in particular, $\left.f_{1 / 2}(1)=1 / 2\right)$;
(ii) $f_{\alpha}\left(c_{\alpha}\right)=1$
(in particular, $f_{1 / 2}(1 / 2)=1$ ) ;
(iii)
$\left[f_{\alpha}(x)-f_{\alpha}(y)\right] /(x-y) \geq \lambda_{1}$ for all $1 / 2-\delta \leq y<x \leq c_{\alpha}$;
(iv) $\left[f_{\alpha}(x)-f_{\alpha}(y)\right] /(x-y) \leq-\lambda_{2}$ for all $c_{\alpha} \leq y<x \leq 1 / 2+\delta$;
(v) $\left[f_{\alpha}(x)-f_{\alpha}(y)\right] /(x-y) \leq-\lambda_{3}$ for all $1-\delta \leq y<x \leq 1$.

Then the following hold.

(1) If $\min \left\{\lambda_{1} \lambda_{3}, \lambda_{2} \lambda_{3}\right\} \geq[(1+\sqrt{5}) / 2]^{1 / 2^{n}}$ for some integer $n \geq 0$, then there exists a number $0<\varepsilon<\delta$ such that, for every $\alpha$ with $1 / 2<\alpha<1 / 2+\varepsilon, f_{\alpha}(x)$ has a periodic point of least period $2^{n+1} \cdot 3$ in $\left[f_{\alpha}^{2}(1), 1\right]$.

(2) If $\min \left\{\lambda_{1}^{2} \lambda_{3}^{2}, \lambda_{1} \lambda_{2} \lambda_{3}^{2}\right\} \geq[(1+\sqrt{5}) / 2]^{1 / 2}$ for some integer $n \geq 0$, then there exists a number $0<\varepsilon<\delta$ such that, for every $\alpha$ with $1 / 2<\alpha<1 / 2+\varepsilon, f_{\alpha}(x)$ has a periodic point of least period $2^{n+2} \cdot 3$ in $\left[f_{\alpha}^{2}(1), 1\right]$.

Remark 6. In the above corollary, the family $f_{\alpha}(x)$ is quite arbitrary and may already have, for every $\alpha$ with $0 \leq \alpha \leq 1$, lots of periodic points of any periods. However, since $\{1 / 2,1\}$ is a periodic orbit of $f_{\frac{1}{2}}(x)$ with least period 2, our result guarantees that the family $f_{\alpha}(x)$ does have a bifurcation at $\alpha=1 / 2$ from the period 2 orbit $\{1 / 2,1\}$ directly to periodic points of least period $2^{m+1} \cdot 3$ for some integer $m \geq 0$. This, in turn, implies that the family $f_{\alpha}^{2}(x)$ has a bifurcation at $\alpha=1 / 2$ from each of the fixed points $x=1 / 2$ and $x=1$ directly to periodic points of least period $2^{m} \cdot 3$. In the following, we give an explicit $f_{\alpha}(x)$ which also has a series of reverse bifurcations.

COROLLARY 5. Let $\delta, \lambda_{1}, \lambda_{2}$, and $\lambda_{3}$ be four fixed positive numbers with $0<\delta<1 / 2, \lambda_{1} \lambda_{3} \geq 8$, and $\lambda_{2} \lambda_{3} \geq 8$. Let $f(x)$ be a continuous map in $C^{0}(I, I)$ which satisfies the following five conditions:

(i) $f(x)$ is increasing on $[0,1 / 2]$ and decreasing on $[1 / 2,1]$; 
(ii) $3 / 4 \leq f(0), f(3 / 4) \leq 1 / 2$, and $f(1)=0$;

(iii) $[f(x)-f(y)] /(x-y) \geq \lambda_{1}$ for all $1 / 2-\delta \leq y<x \leq 1 / 2$;

(iv) $[f(x)-f(y)] /(x-y) \leq-\lambda_{2}$ for all $1 / 2 \leq y<x \leq 1 / 2+\delta$;

(v) $[f(x)-f(y)] /(x-y) \leq-\lambda_{3}$ for all $1-\delta \leq y<x \leq 1$.

Let $f_{\alpha}(x)=1-\alpha[1-f(x)]$ for $0 \leq x \leq 1$ and $0 \leq \alpha \leq 1$. Then the following hold.

(1) For $0 \leq \alpha \leq 1 / 2, f_{\alpha}(x)$ has no periodic points other than fixed points and periodic points of least period 2 .

(2) There exists a positive number $\varepsilon<\delta$ such that, for every $\alpha$ with $1 / 2<\alpha<1 / 2+\varepsilon, f_{\alpha}(x)$ has a periodic point of least period 6 in $\left[f_{\alpha}^{2}(1), 1\right]$.

(3) For $\alpha=1, f_{\alpha}(x)$ has no periodic points with least period strictly greater than 4 , but it has periodic points of least period 2 .

Remark 7. If $f_{\alpha}(x)$ is defined as in the above corollary, then the one-parameter family $f_{\alpha}^{4}(x)$ of continuous maps in $C^{0}(I, I)$ has only fixed points and no other periodic points for all $0 \leq \alpha \leq 1 / 2$ and for $\alpha=1$. However, for every $\alpha$ with $1 / 2<\alpha<1 / 2+\varepsilon, f_{\alpha}^{4}(x)$ has periodic points of least period 3. Therefore, this family $f_{\alpha}^{4}(x)$ has a direct bifurcation from fixed points to period 3 points at $\alpha=1 / 2$ together with a series of reverse bifurcations from period 3 points back to fixed points as $\alpha$ varies from 0 to 1 .

Remark 8. It is well-known that continuous maps in $C^{0}(I, I)$ without periodic points other than fixed points have very simple dynamics ([3], [4, Theorem 2]) and those in $C^{0}(I, I)$ with periodic points of least period 3 have an uncountable scrambled set on which the dynamics are extremely complicated ([8], see also [5]). Therefore, the family $f_{\alpha}^{4}(x)$ with $f_{\alpha}(x)$ defined as in the above corollary provides examples of oneparameter families of continuous maps in $C^{0}(I, I)$ which have a bifurcation fxom very simple dynamics directly to chaos (see also [6]). 
Remark 9. It is also well-known that continuous maps in $C^{0}(I, I)$ without periodic points of least period $2^{m} \cdot n$ for any integer $m \geq 0$ and any odd integer $n>1$ have zero topological entropy [11] and those in $C^{0}(I, I)$ with periodic points of least period 6 have topological entropy $\geq(\log [(1+\sqrt{5}) / 2]) / 2$ (see $[1],[7])$. Therefore, if $f_{\alpha}(x)$ is defined as in the above corollary, then the topological entropy of $f_{\alpha}(x)$ jumps from zero to some positive number $\geq(\log [(1+\sqrt{5}) / 2]) / 2$ at $\alpha=1 / 2$ and then changes back to zero as $\alpha$ varies from 0 to 1 . since $f_{\alpha}(x)$ is conjugate to the family $g_{\alpha}(x)=\alpha[1-f(1-x)]$ through the function $h(x)=1-x$ (that is $h^{-1} f_{x} h=g_{\alpha}$ ), we see that the topological entropy of the family $g_{\alpha}(x)$ can be strictly decreasing for some values of the parameter.

\section{Proof of Theorem 1}

The proof of Theorem 1 is more or less the same as that in [2]. The major difference is that we use a stronger lemma to get a better estimate at one crucial step. The lemma on which we base our proof of Theorem 1 is Lemma 2 below. This lemma is a trivial consequence of the following easy, but fundamental, result.

LEMMA 1. Let $g \in C^{0}(I, I)$ and let $I_{j}, j=0,1, \ldots m$, be closed subintervals of $I$ such that $I_{j+1} \subset g\left(I_{j}\right)$ for all $j=0,1, \ldots, m-1$, and $I_{m}=I_{0}$. Then there is a point $x_{0} \in I_{0}$ such that $g^{j}\left(x_{0}\right) \in I_{j}$ for all $j=0,1,2, \ldots, m-1$, and $g^{m}\left(x_{0}\right)=x_{0}$.

LEMMA 2. Suppose $g \in C^{\circ}(I, I)$. If there exist two closed subintervals $J$ and $K$ of $I$ such that

(i) $g(J) \supset K$;

(ii) $g(K) \supset J \cup K ;$ and

(iii) $g^{2}(J \cap K) \cap K=\phi$,

then $g$ has a periodic point of least period 3 .

The next lemma is a slight variation of Lemma 3 in [2]. This lemma can also be proved by using Lemma 2 above. 
LEMMA 3. Let $f \in C^{0}(T, I)$ be an expanding mop. Suppose that there exists a point $z$ with $f(z)<z<f^{2}(z)$ such that $f$ is decreasing on $[f(z), z]$ and increasing on $\left[z, f^{2}(z)\right]$. Then either $f$ has a periodic point of least period 6 or if $g=f^{2}$, then there exists a point $w$ with $f(z)<w<z$ such that $f(z)=g(w)<w<g^{2}(w)<z$ and, $g$ is decreasing on $[g(w), w]$ and increasing on $\left[w, g^{2}(w)\right]$.

The following lemma is taken from the proof of the main theorem in [2]. Again, this lemma can also be proved by using Lemma 2 above.

LEMMA 4. Suppose that $\tau \in C^{0}(I, I)$ is an expanding mop with expanding constant $\lambda>1$ which is increasing on $[0, c]$ and decreasing on $[c, 1]$. If $\tau$ has no periodic points of least period 6 and $f=\tau^{2}$, then $f(c)<c<f^{2}(c)$ and, $f$ is decreasing on $[f(c), c]$ and increasing on $\left[c, f^{2}(c)\right]$.

We now proceed to the proof of Theorem 1 .

Proof of Theorem 1. If $\tau$ has a periodic point of least period 3, we are done. So, we may assume that $n \geq 1$ and $\tau$ has no periodic points of least period 3 . By induction on $n$ and by Lemma 4 , it suffices to consider $\tau^{2^{n-1}}$. By Lemma 3 , we have two cases:

Case 1. $\tau^{2^{n-1}}$ has a periodic point $x_{0}$ of least period 6 .

In this case, it is obvious that $x_{0}$ is also a periodic point of $\tau$ with least period $2^{n} \cdot 3$.

Case 2. There exists a point $w$ for $g=\tau^{2^{n}}$ with $g(w)<w<g^{2}(w)$ such that $g$ is decreasing on $[g(w), w]$ and increasing on $\left[w, g^{2}(w)\right]$. In this case, we have

$$
\left[g^{2}(w)-w\right]+[w-g(w)]=g^{2}(w)-g(w) \geq \lambda^{2^{n}}(w-g(w)) .
$$

So,

$$
g^{2}(w)-w \geq\left(\lambda^{2^{n}}-1\right)(w-g(w))
$$

Thus, 


$$
\begin{aligned}
g^{3}(w)-g(w) & \geq \lambda^{2^{n}}\left(g^{2}(w)-w\right) \geq \lambda^{2^{n}}\left(\lambda^{2^{n}}-1\right)(w-g(w)) \\
& \geq w-g(w) .
\end{aligned}
$$

Consequently, $g^{3}(w) \geq w$. Let $J=\left[w, g^{2}(w)\right]$ and $K=[g(w), w]$. Then Lemma 2 implies that $g$ has a period 3 point and so $\tau$ has a periodic point of least period $2^{n} \cdot 3$.

Therefore, in either case, $\tau$ has a periodic point of least period $2^{n} \cdot 3$. This completes the proof of Theorem 1 .

\section{Proof of Theorem 2}

Let $x_{1}$ denote the unique solution of the equation $f(x)=1 / 2$ in $(1-\delta, 1)$. Then it is clear that

$$
\left[f^{2}(x)-f^{2}(y)\right] /(x-y) \geq \lambda_{2} \lambda_{3} \text { for all } x_{0} \leq y<x \leq x_{1} \text {, }
$$

and

$$
\left[f^{2}(x)-f^{2}(y)\right] /(x-y) \leq-\lambda_{1} \lambda_{3} \text { for all } x_{1} \leq y<x \leq 1 \text {. }
$$

For the proof of part (1), we assume that $f^{2}(1) \geq x_{0}$ and $\min \left\{\lambda_{1} \lambda_{3}, \lambda_{2} \lambda_{3}\right\} \geq[(1+\sqrt{5}) / 2]^{1 / 2^{n}}$. Then $f^{2}(x)$ is an expanding map from $\left[x_{0}, 1\right]$ into itself with expanding constant $\geq[(1+\sqrt{5}) / 2]^{1 / 2^{n}} \cdot$ By Theorem 1, $f^{2}(x)$ has a periodic point of least period $2^{n} \cdot 3$ in $\left[x_{1}, 1\right]$. Therefore, $f(x)$ has a periodic point of least period $2^{n+1} \cdot 3$ in $\left[x_{1}, 1\right]$. This proves part (1).

For the proof of part (2), we assume that $f^{2}(1) \geq x_{0}$ and $\min \left(\lambda_{1}^{2} \lambda_{3}^{2}, \lambda_{1} \lambda_{2} \lambda_{3}^{2}\right\} \geq[(1+\sqrt{5}) / 2]^{1 / 2^{n}}$. Then, in particular, $\lambda_{1} \lambda_{3}>1$. So, there is a unique point $c$ in $\left[x_{1}, 1\right]$ such that $f^{2}(c)=x_{1}$. Since $f^{2}(x)$ is strictly decreasing on $\left[x_{1}, 1\right]$, the equation $f^{2}(x)=x$ has a unique solution $z$ in $\left[x_{1}, 1\right]$. It is clear that $x_{1}<z<c$. It is also clear that

and

$$
\left[f^{4}(x)-f^{4}(y)\right] /(x-y) \geq \lambda_{1}^{2} \lambda_{3}^{2} \text { for all } z \leq y<x \leq c
$$




$$
\left[f^{A}(x)-f^{4}(y)\right] /(x-y) \leq-\lambda_{1} \lambda_{2} \lambda_{3}^{2} \text { for all } c \leq y<x \leq 1 .
$$

If $f^{4}(1) \geq z$, then $f^{4}(x)$ is an expanding map from $[z, 1]$ into itself with expanding constant $\geq[(1+\sqrt{5}) / 2]^{1 / 2^{n}}$ and we can apply Theorem 1 to $f^{4}(x)$ on $[z, 1]$. If $f^{4}(1)<z$, then $f^{4}(x)$ has a periodic point of least period 3 in $[c, 1]$. In either case [12], $f(x)$ has a periodic point of least period $2^{n+2} \cdot 3$ in $[0,1]$. This proves part (2).

The proof of Theorem 2 is now complete.

\section{Proof of Theorem 3}

For any continuous function $g(x)$ in $C^{0}(I, I)$ with a point $x_{0} \in I$ such that $g\left(x_{0}\right)<x_{0}<g^{3}\left(x_{0}\right)$, it is well-known (see the proof of Proposition 2.2 in [10]) that, for every integer $n \geq 3, g(x)$ has periodic points of least period $n$ on one side of the point $x_{0}$, that is, the side which contains the point $g\left(x_{0}\right)$. However, on the other side of $x_{0}, g(x)$ may not have periodic points of the corresponding periods. The next lemma shows that, with one more condition, $g(x)$ will have periodic points of any period $m \geq 2$ on both sides of $x_{0}$.

LEMMA 5. Let $g \in C^{0}(I, I)$ and let $x_{0} \in I$ satisfy $g\left(x_{0}\right)<x_{0}<\min \left\{g^{2}\left(x_{0}\right), g^{3}\left(x_{0}\right)\right\}$. Then, for every integer $m \geq 2, g(x)$ has a periodic orbit of least period $m$ whose intersection with each of the intervals $\left(g\left(x_{0}\right), x_{0}\right)$ and $\left(x_{0}, g^{2}\left(x_{0}\right)\right)$ is not empty.

Proof. Let $K=\left[g\left(x_{0}\right), x_{0}\right]$ and let $J=\left[x_{0}, g^{2}\left(x_{0}\right)\right]$. For every integer $m \geq 2$, let $I_{0}=I_{m}=J$ and let $I_{j}=K$ for all $j=1,2, \ldots, m-1$. Then Lemma 1 implies that $g(x)$ has a periodic point $y$ of least period $m$ such that $y \in J$ and $g(y) \in K$. Since both $y$ and $g(y)$ belong to the same periodic orbit of $g(x)$ with least period $m$, we have the desired result.

We can now prove Theorem 3 .

We will show that $f^{2}(1 / 2)<1 / 2 \leq \min \left\{f^{4}(1 / 2), f^{6}(1 / 2)\right\}$. Let $h(x)$ be any continuous map in $C^{0}(I, I)$ which satisfies the following three 
conditions:

$$
\begin{array}{rlrl}
\text { (i) } & h(x) & =2 x & \text { for all } 1 / 2-\delta \leq x \leq 1 / 2 ; \\
\text { (ii) } & h(x)=-x+3 / 2 & \text { for all } 1 / 2 \leq x \leq 1 / 2+\delta ; \\
\text { (iii) } & h(x)=-x+1+\beta & \text { for all } 1-\delta \leq x \leq 1 .
\end{array}
$$

Then it is clear that the following are true:

$$
\begin{aligned}
& \text { (a) } 1-\delta \leq f(\beta) \leq h(\beta) . \\
& \text { (b) } 1 / 2<1-\beta=h^{2}(\beta)=h(h(\beta)) \leq h(f(\beta)) \leq f(f(\beta))=f^{2}(\beta) \leq 1 / 2+\delta . \\
& \text { (c) } 1 / 2<1-\delta \leq f^{3}(\beta) \leq h\left(f^{2}(\beta)\right) \leq h\left(h^{2}(\beta)\right)=h^{3}(\beta)=\beta+1 / 2 . \\
& \text { (d) } 1 / 2=h^{4}(\beta)=h\left(h^{3}(\beta)\right) \leq h\left(f^{3}(\beta)\right) \leq f\left(f^{3}(\beta)\right)=f^{4}(\beta) .
\end{aligned}
$$

since $\beta=f^{2}(1 / 2), f^{2}(\beta)=f^{4}(1 / 2)$, and $f^{4}(\beta)=f^{6}(1 / 2)$, we have shown that $f^{2}(1 / 2)<1 / 2 \leq \min \left\{f^{4}(1 / 2), f^{6}(1 / 2)\right\}$.

If we let $g(x)=f^{2}(x)$, then $g(1 / 2)<1 / 2 \leq \min \left\{g^{2}(1 / 2), g^{3}(1 / 2)\right\}$. So, by Lemma $5, g(x)$ has a periodic orbit $P$ of least period 3 whose intersection with each of the intervals $(B, 1 / 2)$ and $\left(1 / 2, f^{2}(B)\right)$ is not empty. It is obvious that $P \cup f(P)$ is a periodic orbit of $f(x)$ with least period 6 which intersects each of the intervals $(\beta, 1 / 2)$ and $\left(1 / 2, f^{2}(B)\right)$. This completes the proof of Theorem 3 .

\section{References}

[1] L. Block, J. Guckenheimer, M. Misiurewicz and L.-S. Young, "Periodic points and topological entropy of one dimensional maps", (Lecture Notes in Mathematics, 819 (1980),18-34, Springer-Verlag, New York).

[2] Bill Byers, "Periodic points and chaos for expanding maps of the interval", BulZ. Austral. Math. Soc. 24 (1981), 79-83.

[3] W.A. Coppel, "The solution of equations by iteration", Proc. Comb. Phil. Soc. 51 (1955), 41-43.

[4] Ethan M. Coven and G.A. Hedlund, "Continuous maps of the interval whose periodic points form a closed set", Proc. Amer. Math. Soc. 79 (1980), 127-133.

[5] Bau-Sen Du, "Are chaotic functions really chaotic", BulZ. Austral. Math. Soc. 28 (1983), 53-66. 
[6] Bau-Sen Du, "An example of a bifurcation from fixed points to period 3 points", Nonlinear Anal. (to appear).

[1] Bau-Sen Du, "Topological entropy and chaos of interval maps", Nonlinear Anal. (to appear).

[8] Frederick J. Fuglister, "A note on chaos", J. Combin. Theory Series A 26 (1979), 186-188.

[9] Shunji Ito, Shigeru Tanaka and Hitoshi Nakada, "On unimodal linear transformations and chaos I", Tokyo J. Math. 2 (1979), $222-228$.

[10] Tien-Yien Li, Michal Misiurewicz, Giulio Pianigiani and James A. Yorke, "No division implies chaos", Trans. Amer. Math. Soc. 273 (1982), 191-199.

[11] Michal Misiurewicz, "Horseshoes for mappings of the interval", BuzZ. Acad. Polon. Sci. Ser. Sci. Math. 27 (1979), 167-169.

[12] A.N. Sharkovskii, "Coexistence of cycles of a continuous map of the line into itself", Ukrain. Mat. 2h. 16 (1964), 61-7l.

Institute of Mathematics

Academia Sinica

Taipei, Taiwan 115

Republic of China 